\title{
A lateral distribution function for the radio emission of air showers
}

\author{
A. Nelles ${ }^{* 1,2}$, S. Buitink ${ }^{3}$, A. Corstanje ${ }^{1}$, J.E. Enriquez ${ }^{1}$, H. Falcke ${ }^{1,4,5}$, \\ J.R. Hörandel ${ }^{1,4}$, J.P. Rachen ${ }^{1}$, L. Rossetto ${ }^{1}$, P. Schellart ${ }^{1}$, O. Scholten ${ }^{6,7}$, S. ter \\ Veen $^{1,5}$, S. Thoudam ${ }^{1}$, T.N.G. Trinh ${ }^{6}$
}

1 Department of Astrophysics/IMAPP, Radboud University Nijmegen, P.O. Box 9010, 6500 GL

Nijmegen, The Netherlands

2 Now at: Department of Physics and Astronomy, University of California Irvine, Irvine, CA 92697-4575, USA

3 Astrophysical Institute, Vrije Universiteit Brussel, Pleinlaan 2, 1050 Brussels, Belgium

4 NIKHEF, Science Park Amsterdam, 1098 XG Amsterdam, The Netherlands

5 Netherlands Institute of Radio Astronomy (ASTRON), Postbus 2, 7990 AA Dwingeloo, The

Netherlands

6 KVI-CART, University Groningen, P.O. Box 72, 9700 AB Groningen, The Netherlands

7 Interuniversity Institute for High-Energy, Vrije Universiteit Brussel, Pleinlaan 2, 1050

Brussels, Belgium

E-mail: a.nelles@astro.ru.nl

\begin{abstract}
The international LOFAR radio telescope has been used now for four years to detect air showers. Its high antenna density has allowed us to measure the subtle features of the radio emission of air showers. Together with air shower simulations, these data have been used to model the detected signals. The not rotational symmetric footprint is described by an analytical function with as few as four free parameters. The parameters are related to the position of the shower axis, the energy and the distance to the shower maximum. We will show how this parametrization is used for a fast reconstruction of all relevant air shower parameters and what accuracies are obtained in comparison to a full Monte Carlo simulation. We will furthermore elaborate on the absolute scale of our measurements and the predicted signal strengths.
\end{abstract}

The 34th International Cosmic Ray Conference,

30 July- 6 August, 2015

The Hague, The Netherlands

\footnotetext{
* Speaker.
} 

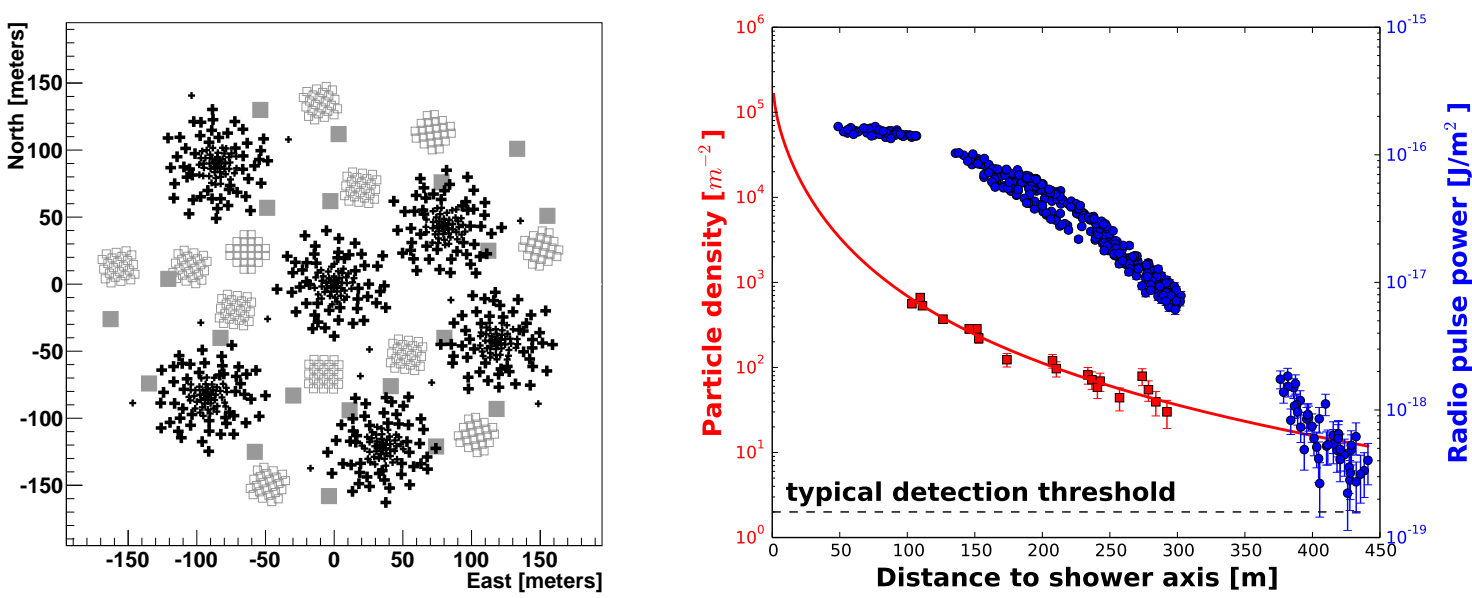

Figure 1: Left: The central core of LOFAR. The black crosses indicate the low-band antennas (30-80 $\mathrm{MHz}$ ), the open squares are the high-band antennas (110-240 MHz) and the full squares are the LORA particle detectors. Right: The signals of an air shower as measured with LOFAR as a function of the distance to the shower axis. The left axis belongs to the red squares that depict the measured particle signals, while the blue circle belong to the right axes and show the radio energy density. Also indicated is a typical detection threshold for both particle and radio measurements, which allows to compare the fall-off of the two distributions.

\section{Introduction}

The cosmic ray experiments that study the radio emission of air showers have matured over the last two years $[1,2,3]$ and similar so did the knowledge about the emission. The experiments have provided an understanding of the emission mechanisms [4, 5], the shape of the wavefront [6, 7], the frequency behavior [8] and the sensitivity of the radio emission to the energy and the height of the maximum of the shower $[9,10]$. Modern air shower simulation codes (e.g. [11, 12]) are able to reproduce the measured signatures, however, require a significant amount of computation that scales with the number of antennas to be simulated.

The emission pattern measured on the ground is not rotationally symmetric around the shower axis, which hinders the usage of a function of solely the distance to the shower axis as it is done for the particle content of a shower (see also Figure 1). The interplay of emission of different polarization creates a characteristics bean-shaped pattern in the emission as measured in low-MHz frequencies on the ground. We have developed a function of as few as four parameters that fits the signal distribution and thereby can be used to reconstruct the energy, the distance to the shower maximum and the two components of the axis position on the ground. The function is suitable to be used at any air shower experiment that detects air showers with more than three antennas. The central core of the LOFAR radio telescope (Figure 1) is densely populated with antennas and has allowed us to test the parameterization in great detail and study subtle features in the signal distribution. An absolute calibration has recently been completed [13] and we are able to show an update of our published study of the lateral distribution function [14]. 

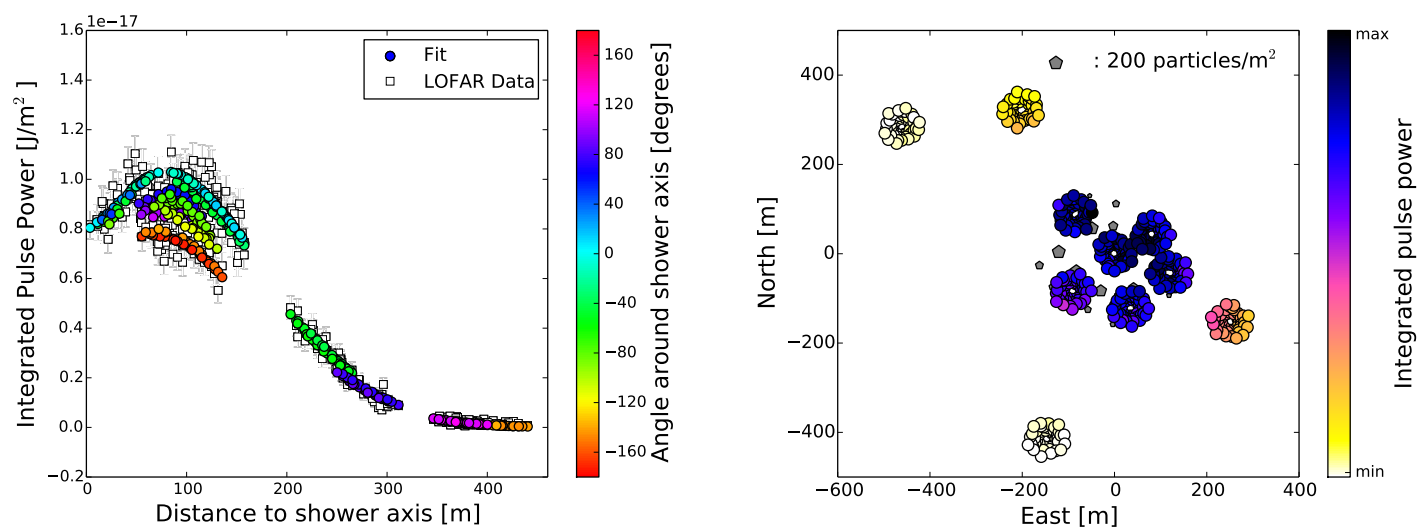

Figure 2: Distribution of integrated signal powers in an air shower as measured with LOFAR. The shower had a zenith angle of $45^{\circ}$ and arrived from north-west with and an energy of $2 \cdot 10^{17} \mathrm{eV}$. Left: Signals as function of distance to the shower axis. Shown are both the measurements, as well as the fit of the lateral distribution as discussed in the text. The reduced $\chi^{2}$ of the fit is 1.05 . The colors indicate the position of the antennas around the shower axis. The structures in the distribution are related to this angle. Right: Distribution of signals in the shower plane spanned by the crossproduct of shower axis, $\vec{v}$, and Earth's magnetic field, $\vec{B}$. The position of the shower axis is at $(0,0)$. The colors of the circles indicate the signal strength. The measured particles are shown by the grey pentagonal markers.

\section{Parameterization and its applicability}

The distribution of integrated pulse powers shows the same characteristic shape of a kidney bean in low-MHz frequencies at various locations on Earth $[14,15,16]$. Guided by this shape, a function based on the sum of two Gaussians (one larger positive one and a smaller negative one) was developed. From the original nine parameters, the function can be reduced to as few as four parameters. At experiments with a high number of antennas, this is not necessary and comes at a cost of a less perfect fit. For LOFAR, the function was reduced to six free parameters, which allows for an optimal fit result.

For the fit, the reconstructed pulses are integrated in time over a window of $55 \mathrm{~ns}$ [17]. They are then summed over all three reconstruction polarisations to obtain a total energy density. Other signal parameters, such as the peak amplitude are also suitable to be fitted with the same function, they are however more influenced by the frequency dependent response of the hardware used. The positions projected into the shower plane in coordinates $x^{\prime}, y^{\prime}$. The fit parameters (2.1) are $X_{c}$ and $Y_{c}$, which relate to the position of the shower axis, $A_{+}$which relates to the energy, $\sigma_{+}$which relates to the distance to the shower maximum and two shape parameters $C_{0}$ and $x_{-}$. The parameters $C_{1}$ and $C_{2}$ are fixed from simulations [18]. In experiments with fewer antennas, it is recommended to first fix $C_{0}$.

$$
\begin{aligned}
P\left(x^{\prime}, y^{\prime}\right)= & A_{+} \cdot \exp \left(\frac{-\left[\left(x^{\prime}-X_{c}\right)^{2}+\left(y^{\prime}-Y_{c}\right)^{2}\right]}{\sigma_{+}^{2}}\right) \\
& -C_{0} \cdot A_{+} \cdot \exp \left(\frac{-\left[\left(x^{\prime}-\left(X_{c}+x_{-}\right)\right)^{2}+\left(\left(y^{\prime}-Y_{c}\right)\right)^{2}\right]}{\left(C_{1} \cdot e^{C_{2} \cdot \sigma_{+}}\right)^{2}}\right) .
\end{aligned}
$$



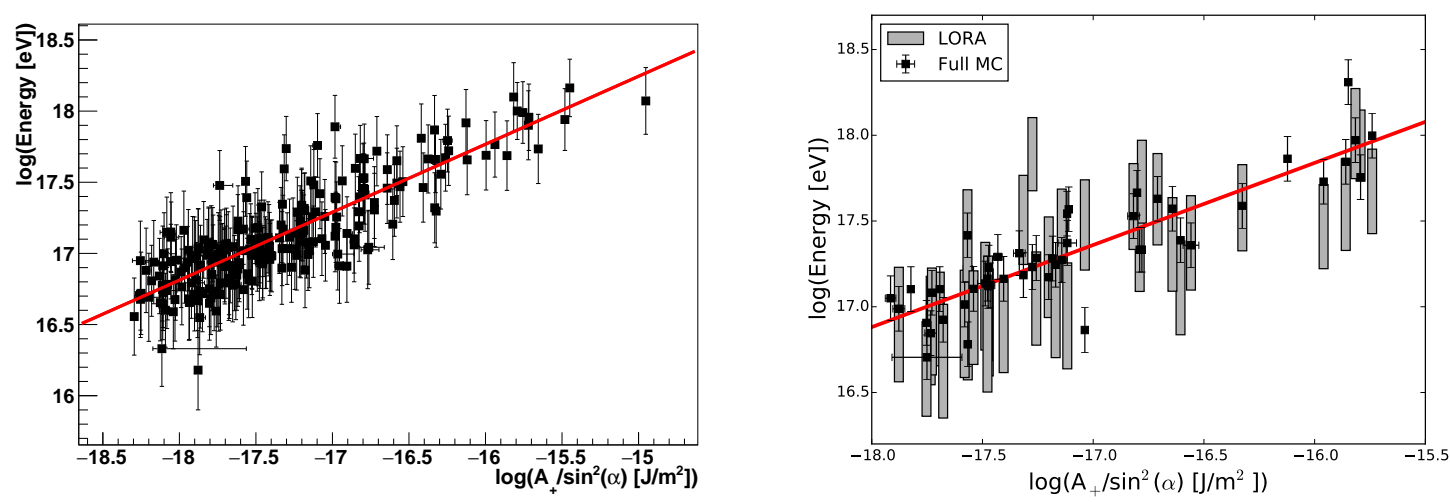

Figure 3: Sensitivity of the fit parameter $A_{+}$to the energy of the air shower. Left: The parameter shows a clear correlation with the energy obtained from the particle detector array LORA. Due to the unknown composition, the energy obtained with the particle detectors is subject to a large uncertainty [21]. The red line indicates a fit of a straight line. The resulting slope is $0.47 \pm 0.03$, which corresponds to a linear scaling of the amplitude with the energy as expected from coherence. Right: For a selected set of air showers, the parameter $A_{+}$is compared to the energy derived with a full Monte-Carlo study and the energy from the particle array LORA. The slope of the best fit of a straight line remains unchanged.

Figure 2 shows the fit of this function to an air shower as measured with LOFAR. The asymmetry is clearly visible, together with the irregular layout of the antennas in the LOFAR core.

\section{Reconstruction of air shower parameters}

In the standard LOFAR reconstruction, the results from the fit of this function are used to determine all essential air shower parameters [17, 14].

\subsection{Shower axis}

The position of the shower axis can be determined at LOFAR, using the particle array, LORA [19], co-located with the central core. The particle array is, however, smaller than the area instrumented with radio antennas, which requires an reconstruction purely based on the radio measurements. As shown in detail in [14] the parameters $X_{c}$ and $Y_{c}$ can be used to reconstruct the position of the shower axis with a resolution comparable to that from the particle detectors, at the core of LOFAR this is about 15 meters. A study of the position of the shower axis shows that it not necessarily coincides with the position of the largest signal strength, which is expected from the asymmetry caused by the interaction of charge excess and geomagnetic emission, as already reported in [20]. Also, the characteristic asymmetry allows us to also reconstruct the shower axis position, if the shower has fallen outside of the instrumented area.

\subsection{Energy}

There are several possibilities to obtain the energy of the shower. The Pierre Auger Collaboration uses the integral over the function in the shower plane to correlate it to the energy of the shower [15]. At LOFAR, it was chosen to study the direct correlation of the parameter $A_{+}$with 

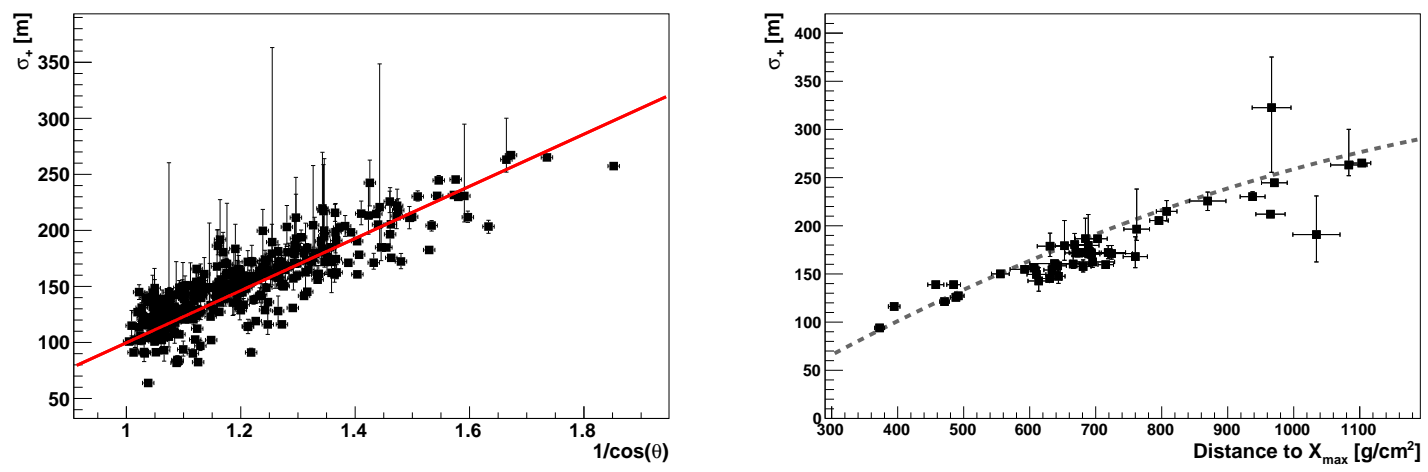

Figure 4: Sensitivity of the fit parameter $\sigma_{+}$to the distance to the shower maximum. Left: The parameter shows a clear correlation with the inverse cosine of the zenith angle of the shower as indicated by the fitted straight line (red). The distribution shows the same amount of spread due to varying height of the shower maximum, as expected from simulations. Right: For a selected set of air showers, the results from a full Monte-Carlo study delivering the distance to the shower maximum are compared to the fit parameter $\sigma_{+}$. The dashed line is the relation between the two parameters as expected from simulations.

the energy. The best choice of parameter also depends on the layout of the detector and the height above sea-level.

Since the energy derived from the radio emission solely depends on the electromagnetic cascade of the shower, the energy resolution depends on whether the cascade is fully developed. Since LOFAR is located at sea-level, there are only very few vertical showers that are not fully developed. However, these showers are more likely to trigger the particle detectors. For detectors at higher altitudes there will be more showers that are stopped in their development, which will limit the energy resolution. Furthermore, the layout of the antenna array determines the stability of certain fit parameters. A small correlation can be found between the shape parameters, meaning that in a sparser array dependencies on, for example the energy, can merge into other parameters. In such a case, integrated quantities are found to be better correlated with the shower parameters. Also, the strength of the local geomagnetic field has a influence of the amount of asymmetry as the ratio of geomagnetic emission to charge excess emission changes. Consequently, certain shape parameters have to be established per location on Earth and detector layout, while the function itself remains appropriate.

Figure 3 shows the correlation of the parameter $A_{+}$with the energy of the shower. The achievable energy resolution is currently determined by the resolution of the particle array. In future work, we will combine measurement of the shower maximum from the radio signals with the particle data to obtain a better energy resolution. We expect that the energy resolution inherent to this method to be better than $30 \%$.

\subsection{Height of the shower maximum}

The parameter $\sigma_{+}$is related to the distance to the shower maximum. The distance to the shower maximum is here defined as

$$
D\left(X_{\max }\right)\left[\mathrm{g} / \mathrm{cm}^{2}\right]=X_{\mathrm{atm}}\left[\mathrm{g} / \mathrm{cm}^{2}\right] / \cos (\theta)-X_{\max }\left[\mathrm{g} / \mathrm{cm}^{2}\right],
$$


where $X_{\mathrm{atm}}$ is the vertical column density of the atmosphere and $X_{\max }$ is the depth in the atmosphere where the air shower reaches its maximum of secondary particles. This dependence of $\sigma_{+}$is expected since most of the radio emission is created near shower maximum and the essential quantity is the distance that the emission traverses from its origin.

Figure 4 shows that the relation can be retrieved from data for the main dependence on the zenith angle. The data confirms that there is a strong dependence on the zenith angle. Still, the large scatter on the distribution indicates that there is likely another parameter influencing $\sigma_{+}$. Since there is no independent detector at LOFAR that can measure $X_{\max }$, only a comparison to a full Monte-Carlo study is possible. As shown in [9], the height of the shower maximum can be determined to an accuracy of $17 \mathrm{~g} / \mathrm{cm}^{2}$ for a first set of 50 air showers. Figure 4 shows the comparison of the thus obtained values for $X_{\max }$ to the $\sigma_{+}$obtained with the fit. The correlation is observed like it was predicted and delivers a combined resolution of $38 \mathrm{~g} / \mathrm{cm}^{2}$. This shows that the parametrization is an adequate tool to obtain a fast reconstruction of the shower maximum.

\section{Conclusions}

We have presented a parameterisation for the signal pattern of the radio emission of air showers as function of the position of the shower axis, the energy and the height of shower maximum. Since the radio emission is not rotationally symmetric, a two-dimensional function is required. The function is used at LOFAR to provide a fast first reconstruction of essential parameters. It delivers a good energy and $X_{\max }$-resolution. The function is suitable for all experiments that measure the full electric-field emitted by a shower in more than three antennas.

\section{Acknowledgement}

The LOFAR cosmic ray key science project acknowledges funding from an Advanced Grant of the European Research Council (FP/2007-2013) / ERC Grant Agreement n. 227610. The project has also received funding from the European Research Council (ERC) under the European Union's Horizon 2020 research and innovation programme (grant agreement No 640130). We furthermore acknowledge financial support from FOM, (FOM-project 12PR304) and NWO (VENI grant 639041-130). AN is supported by the DFG (research fellowship NE 2031/1-1).

LOFAR, the Low Frequency Array designed and constructed by ASTRON, has facilities in several countries, that are owned by various parties (each with their own funding sources), and that are collectively operated by the International LOFAR Telescope foundation under a joint scientific policy.

\section{References}

[1] LOFAR Collaboration, M. P. van Haarlem et al., LOFAR: The LOw-Frequency ARray, Astronomy \& Astrophysics 556 (Aug., 2013) A2, [arXiv: 1305.3550].

[2] J. Schulz for the Pierre Auger Collaboration, Status and Prospects of the Auger Engineering Radio Array, Proc. 34th ICRC, The Hague, The Netherlands (2015).

[3] D. Kostunin for the Tunka-Rex Collaboration, The Tunka Radio Extension: two years of air-shower measurements, Proc. 34th ICRC, The Hague, The Netherlands (2015). 
[4] Pierre Auger Collaboration, A. Aab et al., Probing the radio emission from cosmic-ray-induced air showers by polarization measurements, Physical Review D 89 (2014) 052002.

[5] P. Schellart, S. Buitink, A. Corstanje, J. Enriquez, J. Falcke, H. Hörandel, M. Krause, A. Nelles, J. Rachen, O. Scholten, S. ter Veen, S. Thoudam, and T. Trinh, Polarized radio emission from cosmic ray air showers measured with LOFAR, JCAP 10 (Oct., 2014) 14.

[6] LOPES Collaboration, W. D. Apel et al., The wavefront of the radio signal emitted by cosmic ray air showers, JCAP 9 (Sept., 2014) 25, [arXiv: 1404 .3283].

[7] LOFAR Collaboration, A. Corstanje et al., The shape of the radio wavefront of extensive air showers as measured with LOFAR, Astroparticle Physics 61 (2015) 22-31.

[8] LOFAR Collaboration, A. Nelles et al., Measuring a Cherenkov ring in the radio emission from air showers at 110-190 MHz with LOFAR, Astroparticle Physics 65 (2015) 11-21.

[9] S. Buitink, A. Corstanje, J. Enriquez, H. Falcke, J. Hörandel, T. Huege, A. Nelles, J. Rachen, P. Schellart, O. Scholten, S. ter Veen, S. Thoudam, and T. Trinh, A method for high precision reconstruction of air shower Xmax using two-dimensional radio intensity profiles, Physical Review D 90 (Oct., 2014) 082003.

[10] LOPES Collaboration, W. Apel et al., Experimental evidence for the sensitivity of the air-shower radio signal to the longitudinal shower development, Physical Review D 85 (2012), no. 7071101.

[11] T. Huege, M. Ludwig, and C. W. James, Simulating radio emission from air showers with CoREAS, in American Institute of Physics Conference Series (R. Lahmann, T. Eberl, K. Graf, C. James, T. Huege, T. Karg, and R. Nahnhauer, eds.), vol. 1535 of American Institute of Physics Conference Series, pp. 128-132, May, 2013.

[12] J. Alvarez-Muniz, W.R.Carvalho Jr., and E. Zas, Monte Carlo simulations of radio pulses in atmospheric showers using ZHAireS, Astroparticle Physics 35 (2011), no. 6 325-341.

[13] J. Hörandel et al., Calibration of the LOFAR antennas, Proc. 34th ICRC, The Hague, The Netherlands (2015).

[14] A. Nelles, S. Buitink, A. Corstanje, J. E. Enriquez, H. Falcke, J. R. Hörandel, J. P. Rachen, L. Rossetto, P. Schellart, O. Scholten, S. ter Veen, S. Thoudam, and T. N. G. Trinh, The radio emission pattern of air showers as measured with LOFAR - a tool for the reconstruction of the energy and the shower maximum, JCAP 5 (May, 2015) 18, [arXiv:1411.7868].

[15] C. Glaser for the Pierre Auger Collaboration, The Energy Content of Extensive Air Showers in the Radio Frequency Range of 30-80 MHz, Proc. 34th ICRC, The Hague, The Netherlands (2015).

[16] A. Nelles for the ARIANNA Collaboration, Measuring Neutrinos with the ARIANNA Hexagonal Radio Array, Proc. 34th ICRC, The Hague, The Netherlands (2015).

[17] LOFAR Collaboration, P. Schellart et al., Detecting cosmic rays with the LOFAR radio telescope, Astronomy \& Astrophysics 560 (Dec., 2013) A98, [arXiv:1311.1399].

[18] A. Nelles, S. Buitink, H. Falcke, J. R. Hörandel, T. Huege, and P. Schellart, A parameterization for the radio emission of air showers as predicted by CoREAS simulations and applied to LOFAR measurements, Astroparticle Physics 60 (Jan., 2015) 13-24, [arXiv: 1402 . 2872].

[19] S. Thoudam, S. Buitink, A. Corstanje, J. E. Enriquez, H. Falcke, W. Frieswijk, J. R. Hörandel, A. Horneffer, M. Krause, A. Nelles, P. Schellart, O. Scholten, S. ter Veen, and M. van den Akker, LORA: A scintillator array for LOFAR to measure extensive air showers, Nuclear Instruments and Methods in Physics Research A 767 (Dec., 2014) 339-346, [arXiv:1408.4469]. 
[20] A. Bellétoile, R. Dallier, A. Lecacheux, V. Marin, L. Martin, B. Revenu, and D. Torres, Evidence for the charge-excess contribution in air shower radio emission observed by the CODALEMA experiment, Astroparticle Physics 69 (Sept., 2015) 50-60.

[21] S. Thoudam, S. Buitink, A. Corstanje, J. E. Enriquez, H. Falcke, J. R. Hörandel, A. Nelles, J. P. Rachen, L. Rossetto, P. Schellart, O. Scholten, S. ter Veen, T. N. G. Trinh, and L. van Kessel, Measurement of the cosmic-ray energy spectrum above $10^{16} \mathrm{eV}$ with the LOFAR Radboud Air Shower Array, Astroparticle Physics, in press (2015). 